\title{
Right Bundle-branch Block with Left Axis Deviation*
}

\author{
PAUL SALTZMAN, HAROLD LINN, AND ALFRED PICK \\ From the Cardiovascular Institute and the Division of Cardiovascular Disease, Department of Medicine, Michael \\ Reese Hospital and Medical Center, Chicago, Illinois, U.S.A.
}

The electrocardiographic pattern of right bundlebranch block in the præcordial leads associated with left axis deviation in the limb leads has been noted by others. Wilson, Johnston, and Barker (1934) were the first to describe a small wide $R$ and $S$ wave in lead I, with a wide and deep S wave in leads II and III, and a typical RBBB pattern in the præcordial leads. Recently, Lepeschkin (1964) reviewed the various cardiographic and pathological alterations associated with this pattern. There now appears to be general agreement that the basic lesion consists of predominant right bundle-branch block associated with defects in the distribution of the left bundlebranch. Left and right ventricular hypertrophy, as well as infarction and fibrosis of variable location and extent, are frequently present (Lepeschkin, 1964). The present study was undertaken to determine whether additional and more specific information could be obtained under these circumstances by a detailed study of the spatial vectorcardiograph. The results obtained are the basis for a proposed classification of possible clinical utility.

\section{SUBJECTS AND METHODS}

Spatial vectorcardiograms, along with a standard cardiogram (including V4R), were taken in 23 patients on an Electronics for Medicine DR-8 recorder. Patients were selected on the basis of routine analysis of the daily cardiograms performed in the Heart Station, the only criterion being the availability of the subject. The Frank lead system was used, employing the frontal, horizontal, and right sagittal projections. Table I gives a brief résumé of the significant clinical diagnoses in the total group. Of the 23 patients, 21 were men and 2 were women. The age range was 49 to 88 years-the average 69.

Received November 5, 1965.

* Aided by a grant from the National Heart Institute (HE-06375). A preliminary report was presented at the American Heart Association meetings in October 1964.

\section{ResUlts}

Analysis of the 23 vectorcardiograms revealed that the majority fell into two basic patterns, with the rest in a smaller intermediate category that showed mixed features or was otherwise unclassifiable. The characteristic distinguishing features of the two main types, type A and type B, were found in the

TABLE I

SUMMARY OF CASES

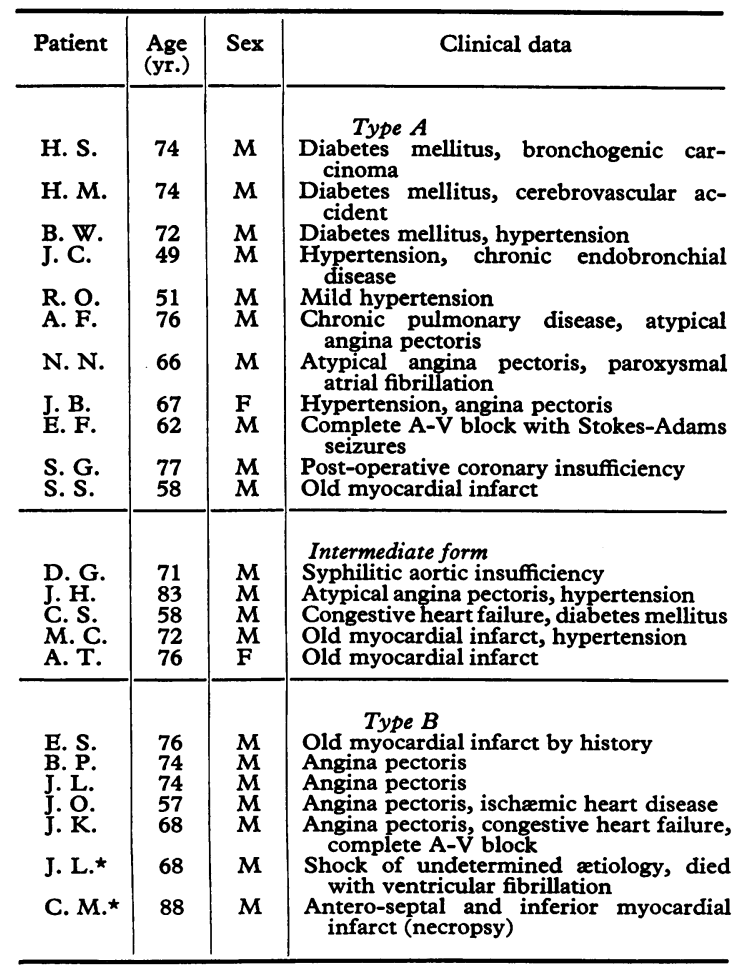


TYPE A

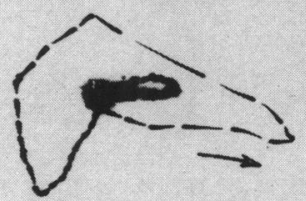

H

$\mathrm{F}$

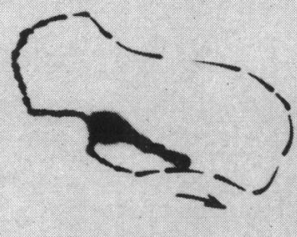

TYPE B

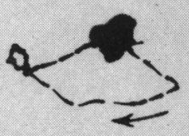

H

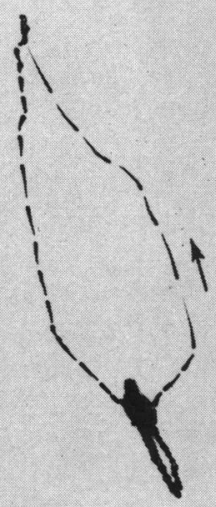

Fig. 1.-Comparison of characteristic vector loops of type A (Case A. F.) and B (Case B. P.) in the horizontal $(H)$ and frontal $(F)$ plane. Note differences in rotation of the horizontal and in the shape and position of the frontal loops. Note in this, as well as in other figures, the direction of movement is toward the narrow part of the interrupted instantaneous vectors.

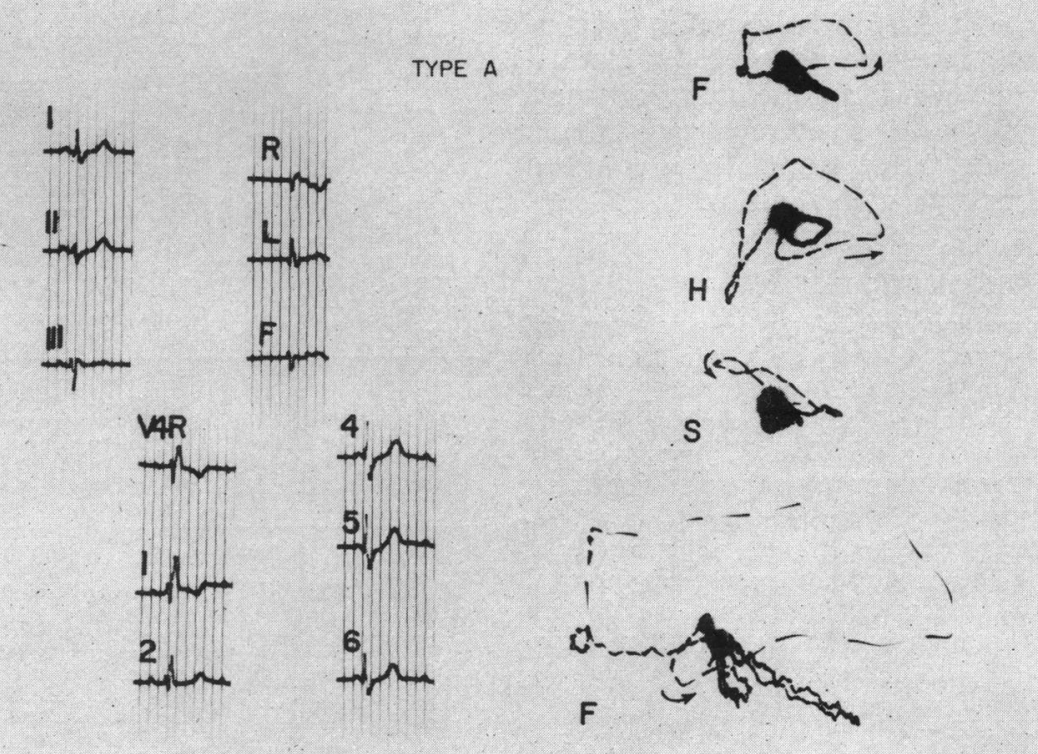

FIG. 2.-Characteristic electrocardiogram and vectorcardiogram in type A (Case E. F.). Note prominent $S$ wave in V4R and V1; counterclockwise rotation of horizontal and frontal loops, the latter flattened (enlarged in lowest panel). 
horizontal and frontal planes and are shown in Fig. 1 and characterized in Table II. Fig. 2 illustrates the typical 3-plane vectorcardiogram, as well as the usual cardiographic pattern of the type A group. The horizontal QRS loop is oriented both anteriorly and posteriorly, often to an equal extent, and rotates in a counterclockwise direction. While the early and mid-portion is essentially normal with initial rightward and anterior forces, and mid-forces directed first leftward and then mainly posteriorly, the pre-terminal and terminal forces are oriented anteriorly and to the right with striking slowing of the inscription. Thus, in the horizontal plane, the loop resembles that seen in uncomplicated RBBB (Braunwald et al., 1956; Scherlis and Lee, 1963).

The frontal QRS loop also showed a characteristic form in group A. The loop always presented early forces directed inferiorly with the main body lying superiorly and rotating in a counterclockwise direction. The over-all shape of this loop was particularly striking as it was flattened and almost rounded in appearance. As expected, the delay was in the pre-terminal and terminal forces. The right sagittal QRS loop, as well as the T loop (as in all vectorcardiograms studied), did not show any characteristic or additionally useful alterations. The cardiogram in type A usually had a prominent $S$ wave in V4R and/or V1, a $Q$ in lead $I$, an initial $R$ in leads II, III, and AVF, and a QRS that was often
TABLE II

VECTORCARDIOGRAPHIC CHARACTERISTICS OF TWO VCG TYPES

\begin{tabular}{l|l|c}
\hline Horizontal & \multicolumn{1}{|c|}{ Type A } & \multicolumn{2}{|c}{ Type B } \\
\cline { 2 - 3 } Frontal & $\begin{array}{l}\text { Antero-posterior, counter- } \\
\text { clockwise } \\
\text { Mainly superior, counter- } \\
\text { clockwise, flat, and } \\
\text { rounded }\end{array}$ & $\begin{array}{c}\text { Mainly anterior, clock- } \\
\text { wise } \\
\text { Mainly superior, counter- } \\
\text { clockwise, elongated }\end{array}$ \\
\hline
\end{tabular}

less widened, the delay being confined to the latter part of the complex.

A total of 10 vectorcardiograms were included in type A. Clinical data (Table I) revealed that 5 patients had no evidence of clinical coronary disease and/or myocardial infarction, though generalized vascular disease was apparent. Significant coronary disease and/or myocardial infarction was possibly present in 3, and definitely found in 2 of the remaining 5 patients.

Fig. 3 illustrates the typical 3 plane vectorcardiographic and cardiographic pattern of type B. The horizontal loop is almost or completely anterior, and apart from a tendency for the early forces to move counterclockwise, shows almost complete clockwise rotation. On occasion, the initial forces were directed posteriorly, the loop then turning anteriorly throughout the remainder of its course. This latter

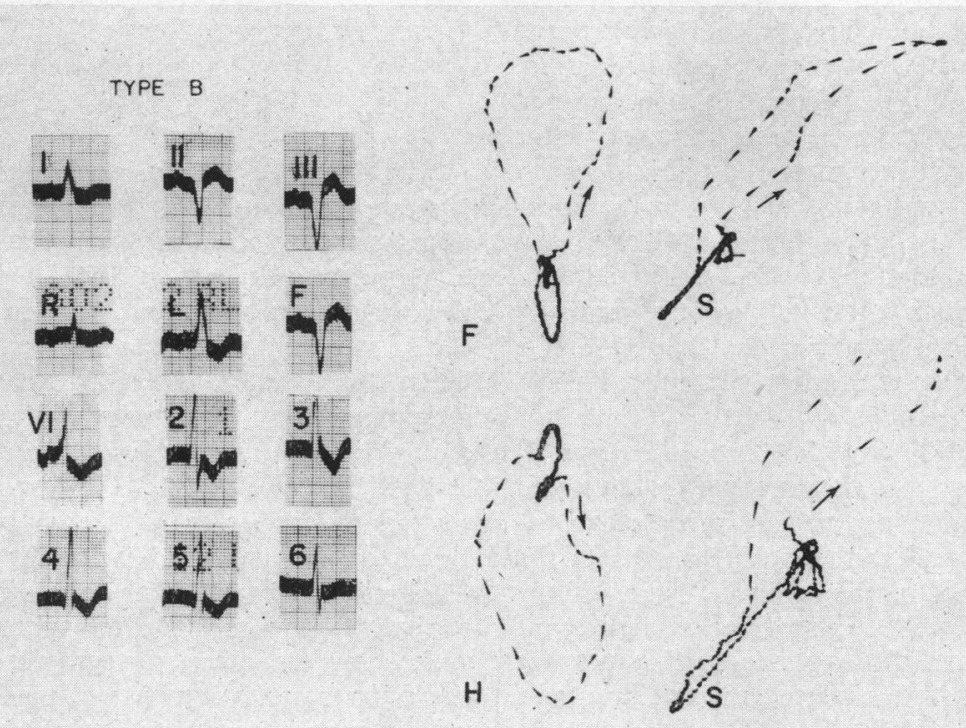

FIG. 3.-Characteristic electrocardiogram and vectorcardiogram in type B (Case J. L.). Note a broader QRS complex with a slurred $\mathrm{R}$ wave in V1; counterclockwise rotation of an elongated superiorly oriented frontal loop and clockwise rotation of a predominantly anterior horizontal loop. 
pattern was invariably present when prominent and slurred $Q$ waves were present across the cardiographic præcordial leads ("anterior wall infarction"). The delay or slowing of the QRS loop was generally more pronounced in all planes and often occurred in the mid, as well as later portions of depolarization.

The frontal loop of type B generally also assumed a typical form. Initial forces were directed either inferiorly or immediately superiorly while the main body was superior and the rotation was counterclockwise. Again, the over-all shape of this loop was its distinctive feature, being elongated and narrow. The cardiogram in this group generally showed a $Q$ wave in V4R and/or V1, and had a broader QRS complex with more generalized slowing compared to type $A$.

Seven vectorcardiograms fell into the type B category (Table I). Six patients had evidence of clinical coronary disease and/or myocardial infarction, and the two cardiovascular deaths in the 23 patients occurred in this group. None of the 7 patients were found to be free of significant coronary disease, and the frequency of important symptoms referable to a diseased myocardium was apparent.

A third, or intermediate, group was also identified (Fig. 4). This group, consisting of 6 patients, had variable patterns, often with mixed characteristics of both type A and B. Others showed a more bizarre configuration and could not be readily classified. The clinical background in this group is seen in Table I. Coronary artery disease was generally present in this mixed group.

\section{Discussion}

Right bundle-branch block with left axis deviation, as pointed out by Lepeschkin (1964), is only one type of electrocardiographic manifestation of bilateral bundle-branch block. In his extensive review, Lepeschkin has classified this particular cardiographic finding into 3 types, $\mathrm{Va}, \mathrm{Vb}$, and $\mathrm{V} 1$. This classification depends upon variable $Q R S$ alterations within the major pattern of a prominent and widened $R$ or $R^{\prime}$ in lead V1 and deep $S$ waves in leads II, III, and AVF. However, the pathological alterations appear to be similar in all three types. Based on detailed studies by Unger et al. (1958), Lenègre (1957), and others, there now appears to be general agreement that this pattern is associated with complete, or nearly complete, interruption of the right bundle-branch and marked involvement, up to nearly complete interruption, of the left bundle-branch. These histological studies are supported by clinical evidence of the frequency of complete heart block in these patients. In addition, 
variable degrees of both left and right ventricular hypertrophy have been described, as well as myocardial infarction, though the latter has been proved in less than half of the reported cases (Lepeschkin, 1964).

In 1954, Richman and Wolff described electrocardiographic and vectorcardiographic studies in 4 patients who were characterized as having "left bundle branch block masquerading as right bundle branch block". As pointed out above, this description does not fit the generally accepted pathological alterations for this entity. More recent vecorcardiographic studies were carried out by a Japanese group (Mashima et al., 1963), but 2 of their 4 patients were young men and, thus, are not comparable to our findings.

Our present classification into three major groups, of which two, usually, have clear and easily definable vectorcardiographic characteristics, appears to offer a simpler and perhaps more useful approach to studies of this abnormality. A summary of the major features seen is presented in Table II.

Type A shows a more normal-appearing vectorcardiogram apart from the superior orientation of a flattened frontal loop (left axis deviation). In addition, the horizontal loop appears essentially normal except for the terminal delay oriented to the right and anteriorly, the pattern described for the typical and uncomplicated RBBB (Braunwald et al., 1956; Scherlis and Lee, 1963). Indeed, the absence of clinically apparent coronary artery disease in $\mathbf{5}$ of the 10 patients in this group, along with the frequently insignificant cardiac findings, i.e. normal heart size, suggest a better prognosis for the patient when type $A$ is present.

On the other hand, the type B loop shows considerable deviation from the normal, as well as from that ordinarily encountered in patients with RBBB. There is usually greater delay which involves most of depolarization rather than being limited to the pre-terminal and terminal forces. Even more apparent is the narrow and elongated frontal loop which, on occasion, shows initial forces directed immediately superiorly. Also, the horizontal loop is completely, or almost completely, anterior in direction and clockwise in rotation. The frequency of overt and symptomatic coronary disease in patients with type $B$, along with the frequently associated cardiomegaly, congestive heart failure, and death is striking, and suggests a generally poorer prognosis as compared to patients with the type A form.

The significance of the intermediate group is, so far, not clear, though it is tempting to consider this form to be in transition between type A and type B, and thus indicate progressing myocardial damage.
The precise pathological differences that could account for these differing vectorcardiogram patterns are unknown but deserve further study. Although characteristically attributed to right ventricular hypertrophy, a clockwise and anteriorly directed horizontal loop has been described in the presence of RBBB alone (Scherlis and Lee, 1963; Milnor, 1957; Miquel et al., 1958). It is tempting to relate the latter to more extensive alterations in the right bundle-branch (fibrosis and/or infarction), but this requires histological proof. Right ventricular hypertrophy alone may produce the horizontal loop pattern described in type B (Milnor, 1957). Although hypertrophy of the right ventricle has been described in pathological descriptions of the QRS abnormalities that we are concerned with (Lepeschkin, 1964; Unger et al., 1958), such vectorcardiographic alterations are usually encountered with more severe right-sided hæmodynamic changes. Infarction of the true posterior wall of the left ventricle has also been described as giving rise to the type B horizontal loop alteration (Walsh et al., 1962; Hugenholtz, Forkner, and Levine, 1961). It is, thus, possible to indict additional fibrosis and/or infarction in this region as the direct causative factor that accounts for the differences in the horizontal plane between types A and B. Unfortunately, these interpretations are purely speculative and their substantiation must await more refined pathological studies. The meaning of frontal loop variations is still undetermined.

Although the vectorcardiogram appears to delineate more clearly the described types, standard cardiographic analysis offers similar, if less precise, indications as to whether type A or type $B$ will be present. Thus, absence of a $Q$ wave with a prominent $S$ wave in V4R and/or V1 indicates type $A$. This interpretation is supported by a slightly widened QRS complex showing its delay in the latter part of the complex. Alternately, a $Q$ wave is present in V4R and/or V1 in the type B pattern. In addition, a broad QRS showing more diffuse delay may be seen and a QS pattern may be present in leads II, III, and AVF. However, these standard cardiographic findings were not always readily apparent, particularly when V4R was not taken or when rapid paper speeds were not employed.

\section{SUMMARY}

Twenty-three patients with right bundle-branch block and left axis deviation were analysed vectorcardiographically utilizing the Frank lead system. Two basic vectorcardiographic patterns, types $A$ and $B$, were identified, as well as an intermediate group.

In type $\mathrm{A}$, present in 10 patients, the characteristic 
findings were a rounded or flattened frontal loop and a horizontal loop showing the classical features of right bundle-branch block, i.e. terminal anterior and rightward delay with counterclockwise rotation.

Type B, present in 7 cases, showed an elongated, narrow frontal loop. The horizontal loop was oriented predominantly anteriorly and showed clockwise rotation. The delay associated with bundle-branch block was usually more diffuse and pronounced in this type.

These vectorcardiographic findings were correlated with the electrocardiographic alterations as well as the clinical data. The latter suggested more extensive myocardial disease and a poorer prognosis in type $B$ as compared with type A.

\section{REFERENCES}

Braunwald, E., Donoso, E., Sapin, S. O., and Grishman, A. (1956). Right bundle-branch block. Hemodynamic, vectorcardiographic and electrocardiographic observations. Circulation, 13, 866.

Hugenholtz, P. G., Forkner, C. E., and Levine, H. D. (1961). A clinical appraisal of the vectorcardiogram in myocardial infarction. II. The Frank system. Circulation, 24, 825.

Lenègre, J. (1957). Contribution à l'Étude des Blocs de Branche Comportant Notamment les Confrontations Élec- triques et Histologiques. Baillière, Paris. (Arch. Mal. Caur, 50, Suppl. 1.)

Lepeschkin, E. (1964). The electrocardiographic diagnosis of bilateral bundle branch block in relation to heart block. Progr. cardiovasc. Dis., 6, 445.

Mashima, S., Harumi, K., Sato, C., Yanai, Y., and Ueda, H. (1963). Atypical bundle branch block-report of four cases with vectorcardiographic observation. $\mathcal{f} a p$. Heart F., 4, 303.

Milnor, W. R. (1957). Electrocardiogram and vectorcardiogram in right ventricular hypertrophy and right bundlebranch block. Circulation, 16, 348.

Miquel, C., Sodi-Pallares, D., Cisneros, F., Pileggi, F., Medrano, G. A., and Bisteni, A. (1958). Right bundle branch block and right ventricular hypertrophy. Electrocardiographic and vectorcardiographic diagnosis. Amer. F. Cardiol., 1, 57.

Richman, J. L., and Wolff, L. (1954). Left bundle branch block masquerading as right bundle branch block. Amer Heart Y., 47, 383.

Scherlis, L., and Lee, Y. (1963). Transient right bundle branch block. Amer. F. Cardiol., 11, 173.

Unger, P. N., Lesser, M. E., Kugel, V. H., and Lev, $M$. (1958). The concept of "masquerading" bundle branch block. Circulation, 17, 397.

Walsh, T. J., Tiongson, P. M., Stoddard, E. A., and Massie, E. (1962). The vectorcardiographic QRSsE-loop findings in inferoposterior myocardial infarction. Amer. Heart F., $63,516$.

Wilson, F. N., Johnston, F. D., and Barker, P. S. (1934). Electrocardiograms of an unusual type in right bundlebranch block. Amer. Heart F., 9, 472. 